\title{
A simple biological method for detecting streptococcal nicotinamide adenine dinucleotide glycohydrolase
}

\author{
N. E. GREEN \\ From the Department of Microbiology, Worthing Hospital, Worthing, Sussex, UK
}

SUMMARY A biological method for detecting streptococcal nicotinamide adenine dinucleotide glycohydrolase (NADG) is presented, based on its ability to inhibit the growth of Haemophilus parainfluenzae. Three hundred clinical isolates of $\beta$-haemolytic streptococci were tested. All isolates producing NADG belonged to Lancefield's groups A, C, or G.

Streptococcal NADG (NAD'ase, DPN'ase) has been extensively characterised (Carlson et al., 1957) and shown to be an extracellular product of growth with classical enzmic properties. It has been purified and shown to catalyse specifically the cleavage of nicotinamide adenine dinucleotide at the nicotinamide-ribose linkage. NADG does not appear to be inducible since it is formed in a defined minimal medium free of substrate and the addition of substrate does not influence its production (Bernheimer et al., 1957).

Streptococcal NADG has been described as cardiotoxic (Van Heyningen, 1962) and postulated as a leucotoxin distinct from the streptolysins (Bernheimer et al., 1957; Wilson, 1957). Although there is a high correlation between NADG activity and leucotoxicity, subsequent evidence points towards streptolysin $\mathbf{S}$ as the substance responsible for leucocyte destruction (Ofek et al., 1970). It is nevertheless possible that more than one streptococcal product is involved. NADG inhibits the oxidation of citrate, fumarate, and $\alpha$-keto-glutarate by mitochondria (Carlson et al., 1956).

To date, the only microbes found to produce specific NADG are Neurospora crassa, certain strains of streptococci of Lancefield's groups A, C, and G (Bernheimer et al., 1957), and Yersinia pestis (Ajl et al., 1956). A very similar but less specific enzyme, NAD pyrophosphatase, is found in Mycobacterium butyricum, Bacillus subtilis, Proteus morganii, Proteus vulgaris, and Proteus rettgeri but is very unusual in that it requires 'heat activation' (Swartz et al., 1956).

Correlation between production or lack of produc-

Received for publication 5 December 1978 tion of NADG by group A streptococci and their M type has been reported (Lazarides and Bernheimer, 1957). M-types 3, 4, 6, and 12 were found to produce NADG with striking regularity, whereas M-types 1, $5,14,19$, and 24 tended not to produce NADG. Association between NADG production and serum opacity factor (SOF) has also been studied in an attempt to relate them to the organisms $\mathbf{M}$ - and Ttype (Ofek et al., 1971). This provided a close association between serotypes, as tested by $T$ agglutination, and the ability to produce NADG and/or SOF, which provided a simple means of dividing given $T$-patterns into four enzmic combinations.

Originally, NADG was detected by a spectrophotometric method (Bernheimer et al., 1957), which measured residual NAD after incubation of a known amount of substrate with dialysed culture supernatant. These methods required large quantities of culture supernatant (in one method 15 litres), dilutions in buffer, and the use of a water bath, ice bath, and spectrophotometer. Recently, a slightly simpler method has been described (Lütticken et al., 1976) which utilises the fluorescence of NAD under ultraviolet light that develops on the addition of strong alkali. However, this method still requires buffer and culture supernatants in addition to the use of an ice bath, strong alkali, and an ultraviolet light source.

\section{Principle}

The method presented here is much simpler than those previously described. The basis of the test is the fact that the substrate for NADG is also the specific growth-requiring factor for Haemophilus parainfluenzae. Growth of $\boldsymbol{H}$. parainfluenzae on a 
minimal medium with the addition of NAD (V factor) was found to be inhibited by NADGproducing streptococci. Non-producers of NADG had no effect on the growth of $H$. parainfluenzae under the same conditions.

That this inhibition is not due to some non-specific substance produced by certain streptococci which kills $H$. parainfluenzae was indicated by the fact that the zone size was inversely proportional to the substrate concentration.

Three hundred clinical isolates of $\beta$-haemolytic streptococci were examined for NADG production using this biological method.

\section{Material and method}

\section{IDENTIFICATION OF ISOLATES TESTED}

All of the isolates included in this study conformed with the definition of $\beta$-haemolytic streptococci (Cowan and Steel, 1974) in that they were Grampositive, catalase-negative cocci possessing a tendency to form chains and producing zones of $\beta$ haemolysis on Columbia blood agar. ${ }^{1}$ An attempt was made to identify the Lancefield group of each isolate by the Rantz and Randall method (Rantz and Randall, 1955) using anti-sera A-G. ${ }^{2}$

\section{Indicator strain of $\mathrm{H}$. parainfluenzae}

Although clinical isolates of $\boldsymbol{H}$. parainfluenzae were used, two reference strains (NCTC 7857 and NCTC 10665) gave equally satisfactory results. There was no discernible difference between the performance of the capsulated reference strain (NCTC 10665) and the non-capsulated reference strain (NCTC 7857). The indicator strain was maintained on chocolated Columbia blood agar at $37^{\circ} \mathrm{C}$ in an atmosphere containing $5-7 \% \mathrm{CO}_{2}{ }^{3}$ and was subcultured twice weekly.

\section{Performance of the test}

A tryptone soya agar (TSA) plate ${ }^{1}$ was seeded with a suspension of the indicator organism using a sterile cottonwool swab to cover the entire plate. The inoculum size was chosen by trial and error, and optimum results were obtained by emulsifying five to six well isolated colonies of $\boldsymbol{H}$. parainfluenzae in 1-2 ml peptone water. Too light an inoculum caused difficulty in reading, whereas an extremely heavy inoculum still gave readable results, although the zone sizes were usually much reduced. A stab inoculum of a single colony (from an overnight blood

1Oxoid Limited, Wade Road, Basingstoke, Hants. ${ }^{2}$ Wellcome Reagents Limited, Beckenham, Kent.

${ }^{3}$ Hotpack $\mathrm{CO}_{2}$ incubator, Hotpack, Philadelphia, USA. agar purity plate incubated at $37^{\circ} \mathrm{C}$ in a $\mathrm{Gas}^{\mathrm{Pak}}{ }^{4}$ anaerobic jar) of each test strain was then made into the seeded plate. $V$ factor discs $^{5}$ were placed aseptically on the plate, so arranged that each test inoculum was approximately $8-12 \mathrm{~mm}$ from a disc. Plates were incubated at $37^{\circ} \mathrm{C}$ in an atmosphere containing 5-7\% $\mathrm{CO}_{2},{ }^{3}$ and examined the next day for the presence of zones of inhibition of the indicator organism produced by the test strains. Part of a typical test plate is illustrated in the Figure. Provided that the test organism produced a zone of inhibition, it was classified as an NADG producer (zone sizes, under identical conditions, varied from 1 to $15 \mathrm{~mm}$, most being about $5 \mathrm{~mm}$ diameter). If the test organism had no effect on the indicator organism it was classified as a nonproducer of NADG.

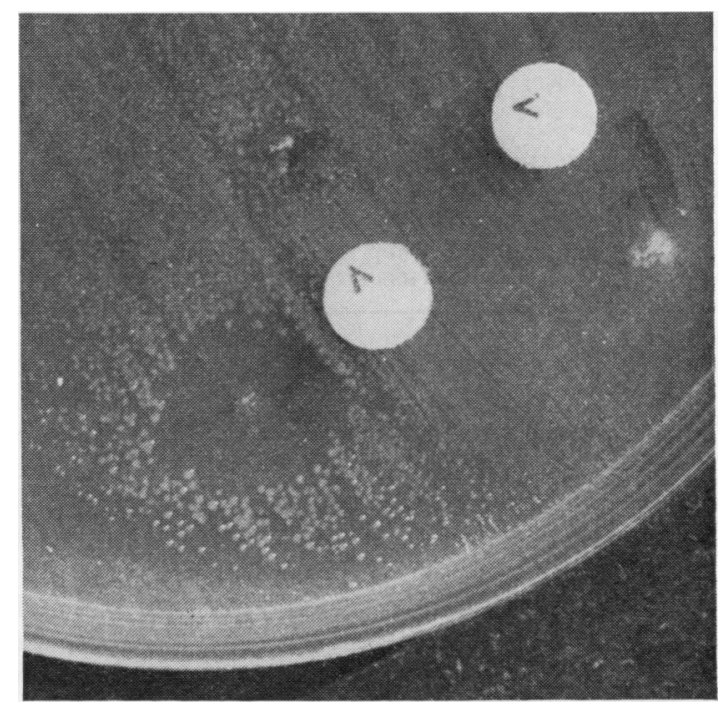

Figure Part of a typical test plate (see text for details) seeded with $\mathrm{H}$. parainfluenzae and the following streptococcal isolates, stab inoculated: (top left) group $A$ ( $+v e)$, (bottom left) group $G(+v e)$, (right) group $D(-v e)$.

Since it is known that streptococci produce NAD, the possibility of omitting the substrate source was investigated. Although a few test organisms produced a zone in this way, the results were variable, some test organisms failing to produce any growth of the indicator organism. Even when a zone was evident, it was only so after prolonged incubation (two or

${ }^{4}$ Gas Pak BBL, B-D Cockeysville, Md 21030, USA.

${ }^{5}$ Mast Laboratories, Liverpool. 
three days). Consequently, $\mathrm{V}$ factor discs were used throughout this study.

Once established, a positive and negative control were included on each test plate.

\section{Results}

The results for the production of NADG by the 300 isolates of $\beta$-haemolytic streptococci are presented in the Table; $84 \%$ of the group $A$ isolates, $58 \%$ of the group $\mathrm{C}$ isolates, and $67 \%$ of the group $\mathrm{G}$ isolates tested produced NADG. There was no NADG producer which did not belong to groups A, C, or G.

Table Results of NADG production by 300 clinical isolates of $\beta$-haemolytic streptococci

\begin{tabular}{llc}
\hline Lancefield group & $\begin{array}{l}\text { No. strains tested } \\
\text { (\% of total) }\end{array}$ & $\begin{array}{l}\text { No. producing NADG } \\
(\%)\end{array}$ \\
\hline A & $93(31)$ & $78(84)$ \\
B & $24(8)$ & $0(0)$ \\
D & $38(13)$ & $22(58)$ \\
E & $9(3)$ & $0(0)$ \\
F & - & $0(0)$ \\
G & $69(23)$ & $46(67)$ \\
No precipitation with & $59(20)$ & $0(0)$ \\
antisera A-G & $300(100)$ & $146(47)$ \\
Totals & & \\
\hline
\end{tabular}

It should be noted that only one method of Lancefield grouping was used, and variation of success rates between various methods has been demonstrated (Broomfield, 1978). By using other methods of Lancefield grouping, the relatively high number of isolates $(20 \%)$ that were not identified as belonging to groups A-G may have been reduced.

Using the described technique, approximately 8-12 tests can be incorporated on one TSA plate. However, if the substrate was included in the medium, up to 20 tests could be accommodated on a single plate. This compares very favourably with the economics of the chemical methods.

The enhancing effect of $1 \%$ horse serum on streptococcal NADG production (Bernheimer et al., 1957) was investigated using 10 positive and 10 negative isolates. A demonstrable effect was not found.

\section{Conclusion and discussion}

When compared with previous results (Bernheimer et al., 1957), an unexpectedly high percentage of the group $A$ isolates tested produced NADG $(84 \%$ in this study, $55 \%$ previously). However, this could be explained by a predominance of particular M-types among the group A streptococci tested.
Compared with the chemical methods for detecting NADG, this biological method is simpler, faster, less laborious, and far less expensive. No dangerous chemicals are involved in this method, but they are in one of the spectrophotometric methods involving cyanide and in the ultraviolet method which utilises strong alkali.

This is believed to be the first time that a biological method for screening streptococcal NADG has been reported. Although this method has not yet been compared with the previously used chemical methods (for example, for sensitivity), it was a success in that the results agreed in essence with previous findings. All the NADG producers belonged to Lancefield groups $A, C$, or $G$, and all the non-NADG producers did not. This biological method may prove useful as a supplement to the $\mathrm{M}$ - and T-typing of group A streptococci.

Thanks are due to Mr D. J. Jefford, AIMLS, for photographic assistance; to Miss R. Aylward for typing the manuscript; and to all the staff of this laboratory for help in supplying test isolates.

\section{References}

Ajl, S. J., Rust, J., Jr., Woebke, J., and Hunter, D. H. (1956). Action of plague toxin on diphosphopyridine nucleotide (Abstract). Federal Proceedings, 15, 581.

Bernheimer, A. W., Lazarides, P. D., and Wilson, A. T. (1957). Diphosphopyridine nucleotidase as an extracellular product of streptococcal growth and its possible relationship to leukotoxicity. Journal of Experimental Medicine, 106, 27-37.

Broomfield, K. J. (1978). An evaluation of streptococcal grouping methods. Medical Laboratory World, 2 (2), 81-86.

Carlson, A. S., Kellner, A., and Bernheimer, A. W. (1956). Selective inhibition by preparations of streptococcal filtrates of the oxidative metabolism of mitochondria procured from rabbit myocardium. Journal of Experimental Medicine, 104, 577-587.

Carlson, A. S., Kellner, A., Bernheimer, A. W., and Freeman, E. B. (1957). A streptococcal enzyme that acts specifically on diphosphopyridine nucleotide. Characterisation of the enzyme and its separation from streptolysin O. Journal of Experimental Medicine, 106, 15-26.

Cowan, S. T. (1974). Cowan and Steel's Manual for the Identification of Medical Bacteria, 2nd edition, p. 55 . Cambridge University Press, London.

Lazarides, P. D., and Bernheimer, A. W. (1957). Association of production of diphosphopyridine nucleotidase with serological type of group A streptococcus. Journal of Bacteriology, 74, 412-413.

Lütticken, R., Lütticken, D., Johnson, D. R., and Wannamaker, L. W. (1976). Application of a new method for detecting streptococcal nicotinamide 
adenine dinucleotide glycohydrolase to various $\mathbf{M}$ types of Streptococcus pyogenes. Journal of Clinical Microbiology, 3, 533-536.

Ofek, I., Bergner-Rabinowitz, S., and Ginsberg, I. (1970). Oxygen stable hemolysins of group A streptococci VII. The relation of the leukotoxic factor to streptolysin S. Journal of Infectious Diseases, 122, 517-522.

Ofek, I., Fleiderman, S., Bergner-Rabinowitz, S., and Ginsberg, I. (1971). Application of enzyme production properties in serotyping of group A streptococci according to T-type. Applied Microbiology, 22, 748-751.

Rantz, L. A., and Randall, E. (1955). Use of autoclaved extracts of hemolytic streptoccoci for serological grouping. Stanford Medical Bulletin, 13, 290-291.

Swartz, M. N., Kaplan, N. O., and Frech, M. E. (1956). Significance of 'heat-activated' enzymes. Science, 123, 50-53.

Van Heyningen, W. E. (1962). In General Pathology, edited by H. W. Florey, 3rd edition, p. 754. Saunders, Philadelphia.

Wilson, A. T. (1957). The leucotoxic action of streptococci. Journal of Experimental Medicine, 105, 463-483.

Requests for reprints to: N. E. Green, Microbiology Department, Worthing Hospital, Lyndhurst Road, Worthing, Sussex, UK. 